\title{
Liraglutide Improved Cardiometabolic Parameters More in Obese than in Non-obese Patients with Type 2 Diabetes: A Real-World 18-Month Prospective Study
}

\author{
Dragana Nikolic · Angelo M. Patti · Rosaria V. Giglio • \\ Roberta Chianetta - Giuseppa Castellino · Antonio Magán-Fernández • \\ Roberto Citarrella · Nikolaos Papanas · Andrej Janez · Anca Pantea Stoian • \\ Ali A. Rizvi · Manfredi Rizzo
}

Received: January 11, 2022 / Accepted: January 28, 2022 / Published online: February 15, 2022

(c) The Author(s) 2022

\begin{abstract}
Introduction: The glucagon-like peptide-1 agonist (GLP1-RA) liraglutide is currently approved for the treatment of both obesity and type 2 diabetes (T2DM). We investigated whether the effect of this agent on cardiometabolic parameters in subjects with T2DM varied in relation to the concomitant presence of obesity. Methods: One hundred thirty-five subjects (78 men and 57 women; age: $62 \pm 10$ years) naïve to incretin-based therapies were treated with
\end{abstract}

Dragana Nikolic, Angelo M. Patti and Rosaria V. Giglio contributed equally to the present work.

D. Nikolic - A. M. Patti - R. V. Giglio ·

R. Chianetta · G. Castellino · A. Magán-Fernández .

R. Citarrella $\cdot$ M. Rizzo $(\bowtie)$

Department of Health Promotion, Mother and

Child Care, Internal Medicine and Medical

Specialties (Promise), University of Palermo,

Palermo, Italy

e-mail: manfredi.rizzo@unipa.it

\section{N. Papanas}

Diabetes Centre, Second Department of Internal

Medicine, Democritus University of Thrace,

University Hospital of Alexandroupolis,

Alexandroupolis, Greece

A. Janez

Department of Endocrinology, Diabetes and Metabolic Diseases, Faculty of Medicine, University Medical Centre Ljubljana, University of Ljubljana, Ljubljana, Slovenia low-dose liraglutide (1.2 mg/day) as an add-on to metformin for 18 months. Patients were divided into two subgroups based on their body-mass index (BMI): (a) obese (BMI $\geq 30$ ) and (b) non-obese (BMI <30). Clinical and laboratory analyses were assessed at baseline and every 6 months.

Results: During follow-up, significant improvements were seen in both groups in fasting glycemia, glycated hemoglobin, waist circumference, and carotid intima-media thickness (cIMT), while body weight, BMI, total cholesterol, and low-density lipoprotein cholesterol decreased significantly in obese subjects only. Correlation analysis revealed that changes in subclinical atherosclerosis (assessed

\section{A. P. Stoian · M. Rizzo}

Department of Diabetes, Nutrition and Metabolic Diseases, Carol Davila University of Medicine, Bucharest, Romania

\section{A. A. Rizvi · M. Rizzo}

Division of Endocrinology, Diabetes and

Metabolism, University of South Carolina School of Medicine Columbia, Columbia, SC, USA

\section{A. A. Rizvi}

Department of Medicine, University of Central Florida College of Medicine, Orlando, FL, USA 
by cIMT) were associated with changes in triglycerides $(r=0.488, p<0.0001)$ in the obese group only.

Conclusion: Liraglutide had beneficial actions on glycemic parameters and cardiometabolic risk factors in both non-obese and obese patients with T2DM, with a greater efficacy in the latter. These findings reinforce the benefits of liraglutide for the cardiometabolic outcomes of obese patients with T2DM in the real-world setting. This has critical importance during the current pandemic, since patients with diabetes and obesity are exposed globally to the most severe forms of COVID-19, related complications, and death.

Trial registration: ClinicalTrials.gov identifier, NCT01715428.

Keywords: Cardiovascular risk; Carotid intima-media thickness; Liraglutide; Obesity; Type 2 diabetes mellitus; Incretins; GLP-1

\section{Key Summary Points}

Type 2 diabetes (T2DM) and obesity require additional interventions in order to have proper cardiometabolic benefit.

The glucagon-like peptide-1 agonist (GLP1-RA) liraglutide is currently approved for the treatment of both obesity and type- 2 diabetes (T2DM).

We investigated, in a real-world setting, whether the effect of liraglutide on cardiometabolic parameters in subjects with T2DM varied in relation to the concomitant presence of obesity.

We found that liraglutide had beneficial actions on glycemic parameters and cardiometabolic risk factors in both nonobese and obese patients with T2DM, with a greater efficacy in the latter.

These findings reinforce the benefits of liraglutide for the cardiometabolic outcomes of obese patients with T2DM in the real-world setting.

\section{INTRODUCTION}

Diabetes mellitus is a highly prevalent metabolic disorder that affects approximately 422 million people worldwide and contributes to 1.6 million deaths each year; further, the incidence of type 2 diabetes mellitus (T2DM) continues to rise, creating a major public health challenge globally [1]. Patients with T2DM have a shorter life expectancy and a higher mortality compared to the general population, mainly due to an increased cardiovascular disease risk [2]; concurrently, there has been a relentless increase in the prevalence of obesity, which is associated with a wide range of comorbidities, including diabetes [3]. Due to the overlapping relationship between insulin resistance, diabetes, and obesity, several antidiabetic medications have been evaluated as potential treatments for these disorders $[4,5]$.

In the last decade, the role of glucagon-like peptide-1 receptor agonists (GLP-1RAs) has been extensively investigated in the treatment of both T2DM and obesity. It was demonstrated that GLP-1RAs could be safely used for the treatment of T2DM, reducing fasting and postprandial hyperglycemia through the regulation of pancreatic beta cells in a glucose-dependent manner [6]. Certain GLP-1RAs, including liraglutide, have also shown beneficial effects on several cardiometabolic parameters [7], as well as efficacy in reducing cardiovascular events and mortality [8], due to their demonstrated anti-atherosclerotic and anti-inflammatory mechanisms [9]. In addition, the role of GLP-1RAs in overweight and obese subjects has been studied extensively. Liraglutide and semaglutide are currently approved treatments for obesity, independently of the presence of T2DM [10].

Liraglutide is prescribed for the treatment of T2DM at dosages of 1.2 and $1.8 \mathrm{mg} / \mathrm{day}$; for obesity, the dosage is up to $3.0 \mathrm{mg} /$ day. These therapeutic indications are based largely on the data obtained from the Satiety and Clinical Adiposity Liraglutide Evidence in Non-diabetic and Diabetic Individuals (SCALE) studies $[11,12]$. The use of liraglutide was demonstrated to have a beneficial effect on 
cardiovascular outcomes, as reported in the landmark Liraglutide Effect and Action in Diabetes: Evaluation of Cardiovascular Outcome Results-A Long Term Evaluation (LEADER) study [13].

Randomized clinical trials usually provide detailed information in highly selected categories of patients who are closely treated and followed over time. However, clinical data from real-world settings are gaining more interest, since they may be more applicable to daily practice [14]. Novel anti-diabetic agents have been extensively evaluated over the last decade in randomized clinical trials, but data from realworld settings are relatively scarce. This may be the reason for the limited adoption of these agents in daily practice, in spite of strong recommendations for their use in current international scientific guidelines $[15,16]$.

We evaluated the real-world effect of liraglutide on metabolic parameters over an 18-month treatment period by comparing obese vs non-obese patients with T2DM treated with the lowest approved dosage of liraglutide $(1.2 \mathrm{mg} /$ day). We chose the lowest approved dosage since it has not been investigated to a large degree in clinical studies in spite of the fact that many patients receive such doses for long periods of time for their glyco-metabolic benefit and due to the lack of significant side effects.

\section{METHODS}

\section{Study Protocol}

A total of 135 patients (78 men, 57 women; mean age $62 \pm 10$ ) with T2DM were enrolled in a perspective study for 18 months. Patients were consecutively recruited from the Unit of Diabetes and Cardiovascular Prevention, University Hospital of Palermo, Italy. The study protocol and all amendments were in agreement with the Helsinki Declaration and good clinical practices, and were reviewed and approved by the Ethics Committee of the University Hospital of Palermo, Italy. This study was registered on clinicaltrials.gov (NCT01715428), and all patients gave their written informed consent to participate. Inclusion criteria were the following: presence of T2DM, age between 18 and 75 years old, monotherapy with metformin, and $\mathrm{HbA} 1 \mathrm{c}$ levels greater than $8 \%$. Exclusion criteria included clinical evidence of severe liver dysfunction or renal failure, previous major cardiovascular events, heart failure, and acute infections such as human immunodeficiency virus, hepatitis $\mathrm{B}$ or $\mathrm{C}$ virus, cancer, or the presence of any neoplasm.

BMI was calculated by dividing body weight $(\mathrm{kg})$ by height $\left(\mathrm{m}^{2}\right)$. The participants were divided into two subgroups: (1) obese patients with $\mathrm{BMI} \geq 30 \mathrm{~kg} / \mathrm{m}^{2}$ and (2) non-obese patients with BMI $<30 \mathrm{~kg} / \mathrm{m}^{2}$. Seventy-two patients in the obese group ( 43 men and 29 women, mean age $61 \pm 10$ years), and 63 patients in the nonobese group (34 men and 29 women, mean age of $63 \pm 9$ years) were enrolled. Clinical and biochemical data were gathered during all patient visits. Liraglutide was administered at a dose of $0.6 \mathrm{mg} /$ day for the first two weeks and then increased to $1.2 \mathrm{mg} /$ day for the entire period of the study in combination with a fixed dose of metformin ranging from 1500 to $3000 \mathrm{mg} /$ day.

Anthropometric data, blood samples, and an ultrasonographic determination of carotid intima-media thickness (cIMT) were recorded at baseline and every 6 months for a total followup period of 18 months. All concomitant therapies, such as antihypertensives, lipid-lowering agents, and aspirin, remained largely unchanged throughout the study in order to minimize potential confounding effects, and the doses of such therapies were stable for at least 4 weeks before enrollment. In addition, all subjects were advised not to change their Mediterranean eating habits and their lifestyle habits (commonly a sedentary lifestyle with little physical activity). Monthly follow-up telephone calls were made in order to ensure no changes in their diet/lifestyle habits as well as their compliance with treatment and to assess for adverse events.

\section{Biochemical Analyses}

Blood samples were collected during the first visit (baseline) and every 6 months. All samples 
were taken after a $14 \mathrm{~h}$ overnight fast. Serum glucose, glycated hemoglobin (HbA1c), total cholesterol, triglyceride (TG), and high-density lipoprotein cholesterol (HDL-C) were measured by routine laboratory methods, while low-density lipoprotein cholesterol (LDL-C) was calculated using the Friedewald formula.

\section{Ultrasonographic Determination of Carotid Intima-Media Thickness}

B-mode real-time ultrasound was performed at baseline and every 6 months in a standardized manner with fixed angles of insonation to evaluate the arterial wall thickness in the carotid arteries as a surrogate marker of subclinical atherosclerosis. The same physician (AMP) prospectively performed all the examinations in a blinded manner, without access to previous scans, using the SonoAce Pico Ultrasound System (Samsung Medison Co., Korea) with a probe of 7.5-10.0 MHz.

Patients were examined in the supine position, and each carotid wall or segment was examined to identify the thickest intimal-medial site. Each scan of the common carotid artery began just above the clavicle, and the transducer was moved to the carotid bifurcation and along the internal carotid artery. Three segments were identified and measured in anterior and posterior planes on each side: the distal $1.0 \mathrm{~cm}$ of the common carotid artery proximal to the bifurcation, the bifurcation itself, and the proximal $1.0 \mathrm{~cm}$ of the internal carotid artery. At each of these sites, we determined the IMT, defined as the distance between the echogenic line representing the intimal blood interface and the outer echogenic line representing the adventitial junction. The maximum carotid IMT value was used for analysis and determined as the mean of the maximum IMT of near-wall and far-wall measurements of both the left-side and right-side arteries for each of the three arterial segments.

For the reliability of the results, we calculated that the coefficient of variation for repeat scans was below $5.0 \%$ for all scans, which was consistent with what we found in previous studies evaluating the effects of different GLP1RAs on the cIMT in T2DM $[7,17]$.

\section{Statistical Analysis}

Statistical analyses were performed using SPSS Statistics for Windows (version 17.0) (IBM, Chicago, IL, USA). All variables were assessed for a normal distribution using the Kolmogorov-Smirnov test. Univariate analysis was performed using the paired $t$ test to estimate the difference between the baseline and the measurements every 6 months, and an analysis of variance (ANOVA) was performed depending on the existence of two or more groups in each comparison. The linear correlation was evaluated with Spearman's test. We also performed multivariate analysis using a multiple regression model in which we included all the investigated parameters that reached statistical significance at univariate analysis in order to assess potential independent predictors of change in cIMT. Data are presented as mean \pm standard deviation for continuous variables and as the percentage for categorical variables. $p<0.05$ was considered to indicate significance.

\section{RESULTS}

The baseline characteristics of all patients included in the study are shown in Table 1 . The two groups of obese and non-obese patients were quite balanced in their anthropometric, biochemical, and clinical data, including drug treatments; indeed, the only statistically significant difference was found for BMI $(p<0.0001$, data not shown).

None of the subjects discontinued metformin or liraglutide, and the liraglutide dose did not need to be reduced during the study since no patients experienced intolerable adverse effects. Mild, transient gastrointestinal symptoms (which mainly occurred during the first two weeks of the study, coinciding with the dosage increment) were experienced in 9 nonobese subjects and in 14 obese subjects (data not shown); in addition, no severe hypoglycemic episodes (as defined by the joint American 
Table 1 Baseline characteristics of all patients included in the study $(n=135)$

\begin{tabular}{lll}
\hline & $\begin{array}{l}\text { Obese patients } \\
(\boldsymbol{n}=72)\end{array}$ & $\begin{array}{l}\text { Non-obese patients } \\
(\boldsymbol{n}=\mathbf{6 3})\end{array}$ \\
\hline Age (years) & $61 \pm 10$ & $63 \pm 9$ \\
Women, $n$ (\%) & $29(40)$ & $29(46)$ \\
Diabetes duration (years) & $9 \pm 7$ & $10 \pm 7$ \\
Smokers, $n$ (\%) & $11(15)$ & $17(27)$ \\
Familial history of cardiovascular disease, $n$ (\%) & $40(58)$ & $32(51)$ \\
Systolic blood pressure (mmHg) & $129 \pm 12$ & $124 \pm 11$ \\
Diastolic blood pressure (mmHg) & $78 \pm 10$ & $75 \pm 8$ \\
Hypertension, $n$ (\%) & $54(75)$ & $43(68)$ \\
Dyslipidemia, $n$ (\%) & $55(76)$ & $47(75)$ \\
Use of antihypertensive drugs & & $9(14)$ \\
Beta-blockers, $n$ (\%) & $16(22)$ & $16(25)$ \\
Angiotensin-converting enzyme inhibitors, $n(\%)$ & $25(35)$ & $8(13)$ \\
Angiotensin II receptor antagonists, $n$ (\%) & $13(18)$ & $7(11)$ \\
Calcium channel blockers, $n$ (\%) & $7(10)$ & $10(16)$ \\
Diuretics, $n$ (\%) & $11(15)$ & $14(22)$ \\
Use of lipid-lowering drugs & & $31(49)$ \\
Statins, $n$ (\%) & $37(51)$ & $2(3)$ \\
Fibrates, $n$ (\%) & $1(1)$ & $8(13)$ \\
Polyunsaturated fatty acids, $n$ (\%) & $7(10)$ & \\
Use of antiplatelet drugs, $n$ (\%) & $19(26)$ & \\
\hline Data are pres) & & \\
\hline
\end{tabular}

Data are presented as mean \pm standard deviation for continuous variables and as number and percentage for categorical variables

Diabetes Association and the Endocrine Society) [18] were recorded.

Clinical and biochemical parameters of the obese patients at baseline and after 18 months of treatment are shown in Table 2 . There was a significant improvement in all investigated parameters after 6 months of treatment. These changes were largely maintained during the 18-month follow-up period. Significant reductions in BMI $(p=0.0425)$, waist circumference $(p=0.0236)$, body weight $(p=0.0471)$, glycemia and HbA1c $(p<0.0001$ for both), total cholesterol $(p=0.0197)$, LDL-C $(p=0.0177)$, and cIMT $(p<0.0001)$ were noted at the conclusion of the study.

The clinical and biochemical parameters of non-obese subjects at baseline and after 18 months of treatment are shown in Table 3. A significant improvement in all the investigated parameters was observed after 6 months of treatment with liraglutide. With the exception of triglycerides and HDL-cholesterol concentrations, significant reductions in waist circumference $(p=0.0067)$, glycemia $(p<0.0001)$, HbA1c $(p<0.0001)$, and cIMT $(p<0.0001)$ were found. 
Table 2 Clinical and biochemical parameters in obese patients before and after follow-up $(n=72)$

\begin{tabular}{lccccr}
\hline & Baseline & 6 months & 12 months & 18 months & p \\
\hline Waist circumference $(\mathrm{cm})$ & $116 \pm 15$ & $113 \pm 12^{*}$ & $112 \pm 12$ & $109 \pm 15$ & 0.0236 \\
Body weight $(\mathrm{kg})$ & $96 \pm 17$ & $91 \pm 16^{*}$ & $91 \pm 17$ & $90 \pm 17$ & 0.0471 \\
Body mass index $\left(\mathrm{kg} / \mathrm{m}^{2}\right)$ & $35 \pm 5$ & $34 \pm 5^{*}$ & $33 \pm 5$ & $33 \pm 5$ & 0.0425 \\
Glycemia $(\mathrm{mmol} / \mathrm{l})$ & $9.6 \pm 3.2$ & $7.4 \pm 2.5^{*}$ & $7.2 \pm 2.1$ & $7.3 \pm 2.7$ & $<0.0001$ \\
HbAlc $(\%)$ & $8.8 \pm 0.9$ & $6.9 \pm 1.4^{*}$ & $6.8 \pm 1.1$ & $6.8 \pm 1.2$ & $<0.0001$ \\
Total cholesterol $(\mathrm{mmol} / \mathrm{l})$ & $4.7 \pm 1.5$ & $4.3 \pm 1.1^{*}$ & $4.2 \pm 0.9$ & $4.2 \pm 1.0$ & 0.0197 \\
Triglycerides $(\mathrm{mmol} / \mathrm{l})$ & $1.9 \pm 0.8$ & $1.6 \pm 0.7^{*}$ & $1.6 \pm 0.9$ & $1.6 \pm 0.8$ & 0.0998 \\
LDL cholesterol $(\mathrm{mmol} / \mathrm{l})$ & $2.7 \pm 1.4$ & $2.4 \pm 0.9^{*}$ & $2.3 \pm 0.9$ & $2.2 \pm 0.9$ & 0.0177 \\
HDL cholesterol $(\mathrm{mmol} / \mathrm{l})$ & $1.14 \pm 0.3$ & $1.19 \pm 0.3^{*}$ & $1.19 \pm 0.3$ & $1.23 \pm 0.3$ & 0.3097 \\
Carotid IMT $(\mathrm{mm})$ & $0.97 \pm 0.17$ & $0.88 \pm 0.13^{*}$ & $0.82 \pm 0.13$ & $0.78 \pm 0.22$ & $<0.0001$ \\
\hline
\end{tabular}

$I M T$ intima-media thickness

${ }^{*}$ Significant difference from baseline after 6 months of treatment $(p<0.05)$

Correlation analysis was performed in order to investigate potential associations between the reduction in subclinical atherosclerosis (assessed by cIMT) and changes in all the investigated parameters. We found a significant correlation between changes in triglycerides and those in cIMT $(r=0.488, p<0.0001)$ in obese subjects only (see Fig. 1). Multiple regression analysis (data not shown) revealed that the only independent predictor of changes in cIMT were those in triglycerides $(p<0.05)$ in the group of obese patients.

Table 3 Clinical and biochemical parameters in non-obese patients before and after follow-up $(n=63)$

\begin{tabular}{lcccrr}
\hline & Baseline & $\mathbf{6}$ months & $\mathbf{1 2}$ months & $\mathbf{1 8}$ months & $\boldsymbol{p}$ \\
\hline Waist circumference $(\mathrm{cm})$ & $97 \pm 9$ & $95 \pm 8^{*}$ & $94 \pm 8$ & $92 \pm 9$ & 0.0067 \\
Body weight $(\mathrm{kg})$ & $72 \pm 10$ & $69 \pm 11^{*}$ & $69 \pm 10$ & $69 \pm 10$ & 0.3190 \\
Body mass index $\left(\mathrm{kg} / \mathrm{m}^{2}\right)$ & $26 \pm 2$ & $25 \pm 3^{*}$ & $25 \pm 3$ & $25 \pm 3$ & 0.1413 \\
Glycemia $(\mathrm{mmol} / \mathrm{l})$ & $9.1 \pm 3.8$ & $8.0 \pm 2.4^{*}$ & $7.4 \pm 2.1$ & $7.1 \pm 2.4$ & $<0.0001$ \\
HbAlc $(\%)$ & $8.8 \pm 0.9$ & $7.15 \pm 1.3^{*}$ & $6.9 \pm 1.0$ & $6.8 \pm 0.9$ & $<0.0001$ \\
Total cholesterol $(\mathrm{mmol} / \mathrm{l})$ & $4.6 \pm 1.3$ & $4.3 \pm 0.9^{*}$ & $4.3 \pm 1.0$ & $4.3 \pm 0.8$ & 0.0518 \\
Triglycerides $(\mathrm{mmol} / \mathrm{l})$ & $1.8 \pm 1.5$ & $1.5 \pm 0.7$ & $1.4 \pm 0.6$ & $1.4 \pm 0.6$ & 0.0912 \\
LDL cholesterol $(\mathrm{mmol} / \mathrm{l})$ & $2.7 \pm 1.2$ & $2.35 \pm 0.8^{*}$ & $2.4 \pm 1.0$ & $2.4 \pm 0.7$ & 0.0998 \\
HDL cholesterol $(\mathrm{mmol} / \mathrm{l})$ & $1.2 \pm 0.3$ & $1.2 \pm 0.3$ & $1.2 \pm 0.3$ & $1.3 \pm 0.3$ & 0.2552 \\
Carotid IMT $(\mathrm{mm})$ & $0.97 \pm 0.18$ & $0.89 \pm 0.14^{*}$ & $0.83 \pm 0.12$ & $0.78 \pm 0.16$ & $<0.0001$ \\
\hline
\end{tabular}

IMT intima-media thickness

*Significant difference from baseline and after 6 months of treatment $(p<0.05)$ 


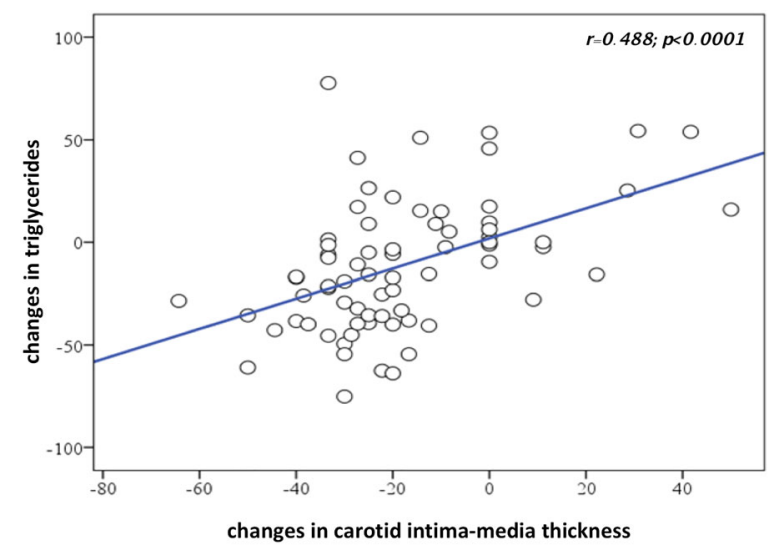

Fig. 1 Correlation plot of the significant association between changes in carotid intima-media thickness and changes in triglycerides after 18 months of liraglutide therapy in obese patients

\section{DISCUSSION}

In this real-world study, 18 months of treatment with liraglutide at the lower approved dose for T2DM of $1.2 \mathrm{mg} /$ day improved several cardiometabolic parameters for primary cardiovascular prevention in both obese and nonobese patients with T2DM. Although there is still no consensus on which GLP-1RA should be used for diabetic patients without a prior cardiovascular event, the American Diabetes Association (ADA) and the European Association for the Study of Diabetes (EASD) have jointly supported liraglutide as the preferred therapeutic option for the treatment of patients with T2DM and atherosclerotic disease [19]. The recommendation was based on the positive cardiovascular outcome shown in the LEADER study with the use of liraglutide [13], as well as on its multiple favorable effects on cardiometabolic risk factors $[8,9]$.

We observed a strong reduction in fasting glycemia and HbA1c in both obese and nonobese patients, in line with the known efficacy of liraglutide in these categories of subjects $[8,11-13]$. The positive findings for anthropometric parameters after only 6 months of liraglutide therapy are also consistent with previous studies, particularly those performed in real-world settings [7]. The reduction in total and LDL cholesterol is consistent with the known actions of liraglutide [7], although this improvement reached statistical significance in the group of obese subjects only. Thus, the low dosage of liraglutide led to a favorable glycometabolic effect in both obese and non-obese subjects with T2DM.

In addition to the demonstration of glycometabolic control, liraglutide exerts a significant benefit in reducing cardiovascular events and mortality [13]. Although the mechanisms involved are still not fully elucidated, increasing evidence suggests that liraglutide is able to reduce cardiovascular risk by directly acting on inflammation and plaque formation and progression [9]. Pre-clinical studies have shown that liraglutide decreases myocardial infarct size by directly acting to reduce intracoronary plaques [20]. There is improved endothelial function, thus inhibiting vascular disease progression, stabilizing atherosclerotic plaques, and lowering inflammation [21]. We also observed a reduction in subclinical atherosclerosis (assessed by cIMT) after liraglutide treatment in both obese and non-obese patients. This has a particular clinical relevance for effective primary cardiovascular prevention measures; indeed, it has been shown in previous studies that endothelial dysfunction and subclinical carotid lesions are key factors in the development of cardiovascular events [22].

An intriguing finding from the present study is that the reduction in cIMT in obese T2DM subjects seems to be mediated by the reduction in triglycerides; this was found in both univariate and multivariate analyses. The development of atherosclerosis is known to be associated with insulin resistance, which is often accompanied by visceral obesity [23]; we found in the present study that liraglutide treatment significantly reduced waist circumference, a measure of visceral obesity, in both groups of obese and non-obese subjects. This reduction in visceral obesity may improve insulin resistance and ultimately result in a reduction in triglyceride; therefore, we cannot exclude that the significant correlation we found between change in cIMT and change in triglycerides in obese patients may be mediated by an improvement in insulin resistance. 
Our finding on the reduction in subclinical atherosclerosis, apparently mediated by the reduction in triglycerides, is in line with the hypothesized direct anti-atherosclerotic action of liraglutide in these patients [9]. Pre-clinical studies in mice models with T2DM have shown that liraglutide's lipid-lowering action is due to its influence on hepatic liposynthesis, which leads to a reduction in triglyceride concentrations [24]. As previously described, both fasting and postprandial triglyceride levels appear to be modulated by liraglutide in patients with T2DM $[25,26]$. It is well known that the triglyceride metabolism plays a central role in cardiovascular risk in T2DM. Longitudinal studies have shown that plasma triglycerides are strong and independent predictors of cardiovascular mortality in patients with T2DM [27]. Subclinical atherosclerosis and coronary artery calcification have also been associated with higher levels of triglycerides in T2DM patients [28]. The accumulation of triglyceride-rich lipoproteins is a key factor in cardiovascular risk [29] since these particles are the precursors of the most atherogenic lipoprotein subfraction, namely small, dense LDL [30]. These particles are an independent risk factor for cardiovascular disease and are predominant in high-risk patients and in certain ethnicities [31-35]; in addition, small, dense LDL are closely associated with pro-inflammatory cytokines, which play a role in both obesity and diabetes [36]. Liraglutide has been shown to reduce small, dense LDL $[37,38]$, in contrast to some traditional antidiabetic agents [39], thus exerting a favorable effect on plaque stability and endothelial dysfunction. Liraglutide also inhibits the formation of foam cells $[20,40]$, which are essentially the deposition of oxidized small, dense LDL in the arteries [9].

The limitations of our study stem mainly from its real-world practice setting. First, the lack of a control group could have hampered the assumption that the observed effect is attributable to liraglutide. In addition, a large number of our patients were treated with concomitant cardiovascular modulating agents such as antihypertensive drugs, statins, and aspirin; this may have influenced the results, although their administration and dosages were largely kept constant during the follow-up. On the other hand, the strengths of our study include the careful clinical and laboratory assessments over a period of 18 months and the blinded 6-monthly evaluation of cIMT, assuring that there was no potential bias. Also, we had a very high rate of adherence to treatment, which was facilitated by the phone calls made to all patients during the follow-up period. Finally, the use of the lower dosage of liraglutide is closer to real-world practice and is of great clinical utility, since randomized clinical trials have focused on the higher dosage of $1.8 \mathrm{mg} /$ day.

\section{CONCLUSIONS}

In conclusion, we report a substantial benefit of liraglutide on several cardiometabolic parameters and surrogate atherosclerotic markers in obese as well as non-obese subjects with T2DM. Interestingly, the results were obtained using a relatively low dose of liraglutide. This study complements the scientific evidence obtained from randomized clinical trials and translates it into a clinical practice setting, confirming the role of liraglutide in reducing subclinical atherosclerosis. Beyond its glucose-lowering action, the impact of this GLP1-RA agent in retarding the genesis and progression of cardiovascular disease in patients with T2DM regardless of their body weight is of considerable interest and deserves further study. This has a particular importance during the current pandemic, since patients with diabetes and obesity have been exposed to the most severe forms of COVID-19, related complications, and death $[41,42]$. Therefore, now more than ever, we need to keep diabetes and obesity under control in patients through proper management.

\section{ACKNOWLEDGEMENTS}

We thank all the participants of the study and Giuseppe Montalto, retired Professor of Internal Medicine, Italy, for valuable advice through the study. 
Funding. No funding or sponsorship was received for this study or publication of this article.

Authorship. All named authors meet the International Committee of Medical Journal Editors (ICMJE) criteria for authorship for this article, take responsibility for the integrity of the work as a whole, and have given their approval for this version to be published.

Author Contributions. Conceptualization: Dragana Nikolic, Angelo M. Patti, Rosaria V. Giglio and Manfredi Rizzo; methodology: Rosaria V. Giglio, Roberta Chianetta, Giuseppa Castellino, Antonio Magán-Fernández, Roberto Citarrella; validation: Dragana Nikolic, Rosaria V. Giglio, Giuseppa Castellino, Antonio MagánFernández, Roberta Chianetta; formal analysis: Dragana Nikolic, Antonio Magán-Fernández, Giuseppa Castellino, Roberta Chianetta; investigation: Dragana Nikolic, Angelo M. Patti, Giuseppa Castellino, Manfredi Rizzo; data curation: Dragana Nikolic; writing-original draft: Dragana Nikolic, Manfredi Rizzo; editing and revision: Nikolaos Papanas, Andrej Janez, Anca Pantea Stoian, Ali A. Rizvi. All authors have read and agreed to the final version of the manuscript.

Disclosures. This manuscript was written independently and the authors did not receive financial or professional help for its preparation. Dragana Nikolic, Angelo M. Patti, Rosaria V. Giglio, Roberta Chianetta, Giuseppa Castellino, Antonio Magán-Fernández, Roberto Citarrella and Ali A. Rizvi have nothing to disclose. Nikolaos Papanas has been an advisory board member of TrigoCare International, Abbott, AstraZeneca, Elpen, MSD, Novartis, Novo Nordisk, Sanofi-Aventis, and Takeda; has participated in sponsored studies by Eli Lilly, MSD, Novo Nordisk, Novartis, and SanofiAventis; received honoraria as a speaker for AstraZeneca, Boehringer-Ingelheim, Eli Lilly, Elpen, Galenica, MSD, Mylan, Novartis, Novo Nordisk, Pfizer, Sanofi-Aventis, Takeda, and Vianex; and attended conferences sponsored by TrigoCare International, AstraZeneca, Boehringer-Ingelheim, Eli Lilly, Novartis, Novo Nordisk,
Pfizer, and Sanofi-Aventis. Andrej Janez discloses consultancies and speaker bureaus for AstraZeneca, Boehringer Ingelheim, Eli Lilly, Merck Sharp \& Dohme (MSD), Novo Nordisk, Sanofi, and Medtronic. Anca Pantea Stoian is currently Vice President of Romanian National Diabetes Committee, and she has given lectures, received honoraria and research support, and participated in conferences, advisory boards and clinical trials sponsored by many companies including Amgen, AstraZeneca, Boehringer Ingelheim, Medtronic, Eli Lilly, Merck, MSD, Novo Nordisk, Novartis, Roche Diagnostics, Servier and Sanofi. Manfredi Rizzo is Editorial Board Member of Diabetes Therapy and former Director, Clinical Medical \& Regulatory Department, Novo Nordisk Europe East and South; he has given lectures, received honoraria and research support, and participated in conferences, advisory boards, and clinical trials sponsored by many pharmaceutical companies including Amgen, Astra Zeneca, Boehringer Ingelheim, Kowa, Eli Lilly, Meda, Mylan, Merck Sharp \& Dohme, Novo Nordisk, Novartis, Roche Diagnostics, Sanofi, and Servier. None of the above-mentioned pharmaceutical companies had any role in this article, which has been written independently, without any financial or professional help, and reflects only the opinion of the authors, without any role of the industry.

Compliance with Ethics Guidelines. The study protocol and all amendments were in agreement with the Helsinki Declaration and good clinical practices and were reviewed and approved by the Ethics Committee of the University Hospital of Palermo, Italy. This study was registered in clinicaltrials.gov (NCT01715428) and all patients gave their written informed consent to participate.

Data Availability. Dragana Nikolic is responsible for the dataset, and may share the information upon reasonable requests.

Open Access. This article is licensed under a Creative Commons Attribution-NonCommercial 4.0 International License, which permits any non-commercial use, sharing, adaptation, 
distribution and reproduction in any medium or format, as long as you give appropriate credit to the original author(s) and the source, provide a link to the Creative Commons licence, and indicate if changes were made. The images or other third party material in this article are included in the article's Creative Commons licence, unless indicated otherwise in a credit line to the material. If material is not included in the article's Creative Commons licence and your intended use is not permitted by statutory regulation or exceeds the permitted use, you will need to obtain permission directly from the copyright holder. To view a copy of this licence, visit http://creativecommons.org/licenses/by$\mathrm{nc} / 4.0 /$.

\section{REFERENCES}

1. World Health Organization. Diabetes. https://www. who.int/health-topics/diabetes. Accessed online on 26 Sept 2021.

2. Lind M, Wedel H, Rosengren A. Excess mortality among persons with type 2 diabetes. N Engl J Med. 2016;374:788-9.

3. World Health Organization. Obesity. https://www. who.int/health-topics/obesity. Accessed online on 26 Sept 2021.

4. Lawson AA, Strong JA, Peattie P, Roscoe P, Gibson A. Comparison of fenfluramine and metformin in treatment of obesity. Lancet. 1970;2:437-41.

5. Nolan JJ, Ludvik B, Beerdsen P, Joyce M, Olefsky J. Improvement in glucose tolerance and insulin resistance in obese subjects treated with troglitazone. N Engl J Med. 1994;331:1188-93.

6. Prasad-Reddy L, Isaacs D. A clinical review of GLP-1 receptor agonists: efficacy and safety in diabetes and beyond. Drugs Context. 2015;4: 212283.

7. Rizzo M, Rizvi AA, Patti AM, Nikolic D, Giglio RV, Castellino G, Li Volti G, Caprio M, Montalto G, Provenzano V, Genovese S, Ceriello A. Liraglutide improves metabolic parameters and carotid intima-media thickness in diabetic patients with the metabolic syndrome: an 18-month prospective study. Cardiovasc Diabetol. 2016;15:162.

8. Nauck MA, Quast DR, Wefers J, Meier JJ. GLP-1 receptor agonists in the treatment of type 2 diabetes-state-of-the-art. Mol Metab. 2021;46: 101102.

9. Rizzo M, Nikolic D, Patti AM, Mannina C, Montalto G, McAdams BS, Rizvi AA, Cosentino F. GLP-1 receptor agonists and reduction of cardiometabolic risk: potential underlying mechanisms. Biochim Biophys Acta Mol Basis Dis. 2018;1864:2814-21.

10. Patel D, Smith A. Patient initiation and maintenance of GLP-1 RAs for treatment of obesity. Expert Rev Clin Pharmacol. 2021;7:1-12.

11. Davies MJ, Bergenstal R, Bode B, Kushner RF, Lewin A, Skjøth TV, Andreasen AH, Jensen CB, DeFronzo RA, NN8022-1922 Study Group. Efficacy of liraglutide for weight loss among patients with type 2 diabetes: the SCALE diabetes randomized clinical trial. JAMA. 2015;314:687-99.

12. Pi-Sunyer $\mathrm{X}$, Astrup A, Fujioka $\mathrm{K}$, Greenway $\mathrm{F}$, Halpern A, Krempf M, Lau DC, le Roux CW, ViolanteOrtiz R, Jensen CB, Wilding JP, SCALE Obesity and Prediabetes NN8022-1839 Study Group. A randomized, controlled trial of $3.0 \mathrm{mg}$ of liraglutide in weight management. N Engl J Med. 2015;373: $11-22$.

13. Marso SP, Daniels GH, Brown-Frandsen K, Kristensen P, Mann JF, Nauck MA, Nissen SE, Pocock S, Poulter NR, Ravn LS, Steinberg WM, Stockner M, Zinman B, Bergenstal RM, Buse JB, LEADER Steering Committee; LEADER Trial Investigators. Liraglutide and cardiovascular outcomes in type 2 diabetes. N Engl J Med. 2016;375:311-22.

14. Mackowiak JI, Mack CD, Irwin DE, Zura R. Randomized clinical trial or real-world evidence: how historical events, public demand, and the resulting laws and regulations shaped the body of medical evidence. J Orthop Trauma. 2021;35:S17-21.

15. Cardio-Metabolic Academy Europe East. Adoption of the ADA/EASD guidelines in 10 Eastern and Southern European countries: Physician survey and good clinical practice recommendations from an international expert panel. Diabetes Res Clin Pract. 2021;172: 108535 .

16. Maranta F, Cianfanelli L, Rizzo M, Cianflone D. Filling the gap between guidelines and real world in the cardiovascular approach to the diabetic patients: the need for a call to action. Int J Cardiol. 2021;329:205-7.

17. Patti AM, Nikolic D, Magan-Fernandez A, Giglio RV, Castellino G, Chianetta R, Citarrella R, Corrado E, Provenzano F, Provenzano V, Montalto G, Rizvi AA, Rizzo M. Exenatide once-weekly improves metabolic parameters, endothelial dysfunction and carotid intima-media thickness in patients with type- 2 
diabetes: an 8-month prospective study. Diabetes Res Clin Pract. 2019;149:163-9.

18. Seaquist ER, Anderson J, Childs B, Cryer P, DagogoJack S, Fish L, Heller SR, Rodriguez H, Rosenzweig J, Vigersky R. Hypoglycemia and diabetes: a report of a workgroup of the American Diabetes Association and the Endocrine Society. Diabetes Care. 2013;36: 1384-95.

19. Davies MJ, D'Alessio DA, Fradkin J, Kernan WN, Mathieu C, Mingrone G, Rossing P, Tsapas A, Wexler DJ, Buse JB. Management of hyperglycemia in type 2 diabetes. A consensus report by the American Diabetes Association (ADA) and the European Association for the Study of Diabetes (EASD). Diabetes Care. 2018;2018(41):2669-701.

20. Noyan-Ashraf MH, Momen MA, Ban K, Sadi AM, Zhou YQ, Riazi AM, Baggio LL, Henkelman RM, Husain M, Drucker DJ. GLP-1R agonist liraglutide activates cytoprotective pathways and improves outcomes after experimental myocardial infarction in mice. Diabetes. 2009;58:975-83.

21. Gaspari T, Welungoda I, Widdop RE, Simpson RW, Dear AE. The GLP-1 receptor agonist liraglutide inhibits progression of vascular disease via effects on atherogenesis, plaque stability and endothelial function in an ApoE(-/-) mouse model. Diabetes Vasc Dis Res. 2013;10:353-60.

22. Corrado E, Rizzo M, Coppola G, Muratori I, Carella M, Novo S. Endothelial dysfunction and carotid lesions are strong predictors of clinical events in patients with early stages of atherosclerosis: a 24-month follow-up study. Coron Artery Dis. 2008;19:139-44.

23. Stepien M, Stepien A, Wlazel RN, Paradowski M, Rizzo M, Banach M, Rysz J. Predictors of insulin resistance in patients with obesity: a pilot study. Angiology. 2014;65:22-30.

24. Cummings BP, Stanhope KL, Graham JL, Baskin DG, Griffen SC, Nilsson C, Sams A, Knudsen LB, Raun K, Havel PJ. Chronic administration of the glucagon-like peptide-1 analog, liraglutide, delays the onset of diabetes and lowers triglycerides in UCD-T2DM rats. Diabetes. 2010;59:2653-61.

25. Hermansen K, Bækdal TA, Düring M, Pietraszek A, Mortensen LS, Jørgensen H, Flint A. Liraglutide suppresses postprandial triglyceride and apolipoprotein B48 elevations after a fat-rich meal in patients with type 2 diabetes: a randomized, double-blind, placebo-controlled, cross-over trial. Diabetes Obes Metab. 2013;15:1040-8.

26. Matikainen N, Söderlund S, Björnson E, Pietiläinen $\mathrm{K}$, Hakkarainen A, Lundbom N, Taskinen MR, Borén J. Liraglutide treatment improves postprandial lipid metabolism and cardiometabolic risk factors in humans with adequately controlled type 2 diabetes: a single-centre randomized controlled study. Diabetes Obes Metab. 2019;21:84-94.

27. Miselli MA, Nora ED, Passaro A, Tomasi F, Zuliani G. Plasma triglycerides predict ten-years all-cause mortality in outpatients with type 2 diabetes mellitus: a longitudinal observational study. Cardiovasc Diabetol. 2014;13:135.

28. Qamar A, Khetarpal SA, Khera AV, Qasim A, Rader DJ, Reilly MP. Plasma apolipoprotein C-III levels, triglycerides, and coronary artery calcification in type 2 diabetics. Arterioscler Thromb Vasc Biol. 2015;35:1880-8.

29. Krauss RM. Atherogenicity of triglyceride-rich lipoproteins. Am J Cardiol. 1998;81:13B-17B.

30. Rizzo M, Berneis K. Who needs to care about small, dense low density lipoproteins? Int J Clin Pract. 2007;61:1949-56.

31. Berneis K, Rizzo M, Hersberger M, Rini GB, Di Fede G, Pepe I, Spinas GA, Carmina E. Atherogenic forms of dyslipidemia in women with polycystic ovary syndrome. Int J Clin Pract. 2009;63:56-62.

32. Bayram F, Kocer D, Gundogan K, Kaya A, Demir O, Coskun R, Sabuncu T, Karaman A, Cesur M, Rizzo M, Toth PP, Gedik V. Prevalence of dyslipidemia and associated risk factors in Turkish adults. J Clin Lipidol. 2014;8:206-16.

33. Rizzo M, Pernice V, Frasheri A, Berneis K. Atherogenic lipoprotein phenotype and LDL size and subclasses in patients with peripheral arterial disease. Atherosclerosis. 2008;197:237-41.

34. Goedecke JH, Utzschneider K, Faulenbach MV, Rizzo M, Berneis K, Spinas GA, Dave J, Levitt NS, Lambert EV, Olsson T, Kahn SE. Ethnic differences in serum lipoproteins and their determinants in South African women. Metabolism. 2010;59: 1341-50.

35. Rizzo M, Spinas GA, Cesur M, Ozbalkan Z, Rini GB, Berneis K. Atherogenic lipoprotein phenotype and LDL size and subclasses in drug-naïve patients with early rheumatoid arthritis. Atherosclerosis. 2009;207:502-6.

36. Abate N, Sallam HS, Rizzo M, Nikolic D, Obradovic $\mathrm{M}$, Bjelogrlic P, Isenovic ER. Resistin: an inflammatory cytokine. Role in cardiovascular diseases, diabetes and the metabolic syndrome. Curr Pharm Des. 2014;20:4961-9.

37. Anholm C, Kumarathurai P, Pedersen LR, Samkani A, Walzem RL, Nielsen OW, Kristiansen OP, Fenger M, Madsbad S, Sajadieh A, Haugaard SB. Liraglutide 
in combination with metformin may improve the atherogenic lipid profile and decrease C-reactive protein level in statin treated obese patients with coronary artery disease and newly diagnosed type 2 diabetes: a randomized trial. Atherosclerosis. 2019;288:60-6.

38. Nikolic D, Giglio RV, Rizvi AA, Patti AM, Montalto G, Maranta F, Cianflone D, Stoian AP, Rizzo M. Liraglutide reduces carotid intima-media thickness by reducing small dense low-density lipoproteins in a real-world setting of patients with type 2 diabetes: a novel anti-atherogenic effect. Diabetes Ther. 2021;12:261-74.

39. Berneis K, Rizzo M, Stettler C, Chappuis B, Braun M, Diem P, Christ ER. Comparative effects of rosiglitazone and pioglitazone on fasting and postprandial low-density lipoprotein size and subclasses in patients with Type 2 diabetes. Expert Opin Pharmacother. 2008;9:343-9.
40. Tashiro Y, Sato K, Watanabe T, Nohtomi K, Terasaki M, Nagashima M, Hirano T. A glucagon-like peptide-1 analog liraglutide suppresses macrophage foam cell formation and atherosclerosis. Peptides. 2014;54:19-26.

41. Stoian AP, Banerjee Y, Rizvi AA, Rizzo M. Diabetes and the COVID-19 pandemic: how insights from recent experience might guide future management. Metab Syndr Relat Disord. 2020;18:173-5.

42. Al Mahmeed W, Al-Rasadi K, Banerjee Y, Ceriello A, Cosentino F, Galia M, Goh SY, Kempler P, Lessan N, Papanas N, Rizvi AA, Santos RD, Stoian AP, Toth PP, Rizzo M, CArdiometabolic Panel of International experts on Syndemic COvid-19 (CAPISCO). Promoting a syndemic approach for cardiometabolic disease management during COVID-19: the CAPISCO international expert panel. Front Cardiovasc Med. 2021;8: 787761. 